
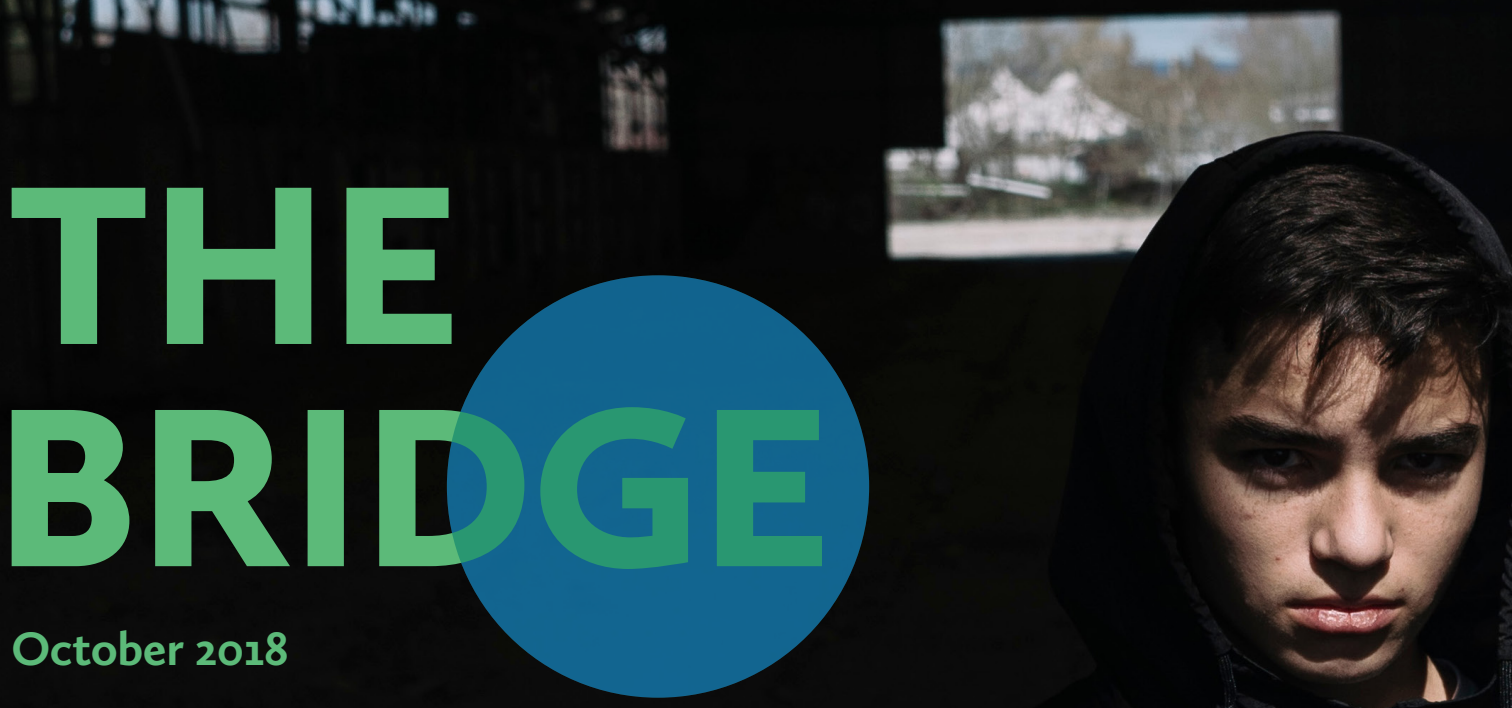

\title{
Forensic Issue
}

Reconstructing child psychopathy

Professor Randall Salekin outlines his key findings

Functional Family Therapy does not reduce ASB or offending in youths.

\section{Also inside \\ Research \\ highlights from our journals JCPP and $\mathrm{CAMH}$}




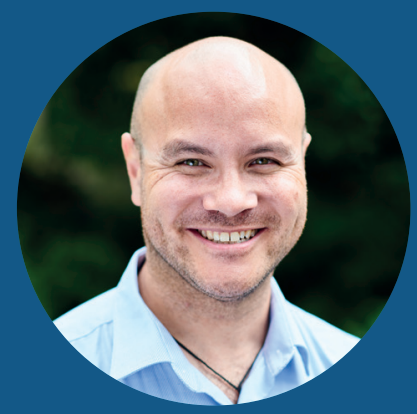

Editorial

\section{Guest Editor, Dr Mark Lovell}

Welcome to the 'Forensics in Children and Adolescents' themed edition of The Bridge, covering the intersection of science, clinical practice and the criminal justice system.

Society has long sought to understand through philosophy and science, what makes people commit crimes or engage in antisocial behaviours. This is a specialist area within child and adolescent mental health services with high variation in service provision despite initiatives at national levels.

The area of forensics is often not well understood within non-forensic services, and the risks involved raise anxieties within clinicians and the multiagency setting as well as for parents and carers. Research is developing in this area, and our understanding of psychological traits and associated other difficulties, e.g. learning, neurodevelopmental disorders and addictions. It is also developing our understanding, through the evidence base, of the costs to individuals and society.

Within this issue, there are summaries based upon journal articles from ACAMH's suite of journals- JCPP and CAMH as well as commissioned pieces on this topic matter giving food for thought.

I hope you find this edition helpful. This pdf has some of the articles from the Forensics issue of The Bridge. Email publications@acamh.org with details of what you'd like to see in future.

\section{Authors}

All research highlights in this issue, except where otherwise mentioned, are written by Dr Jessica Edwards. Jessica is a freelance editor and science writer.

\section{Functional Family} Therapy does not reduce ASB or

\section{offending in youths}

A randomized controlled trial has found that Functional Family Therapy (FFT) plus Management As Usual (MAU) does not significantly reduce youth antisocial behaviour (ASB) or offending compared to MAU alone. The trial, conducted in the UK, recruited 111 youths (aged 10-17 years) through crime prevention agencies such as the Youth Offending Services and Targeted Youth Support Services. All youths had been sentenced for offending or were receiving agency interventions following contact with the police for ASB. The FFT consisted of a range of systemic family therapy interventions plus cognitive, behavioural and social learning theory strategies, which were delivered in 12 sessions across 3-6 months. MAU was delivered by a case worker, usually using a support and counselling model. Interview and questionnairebased assessments were made at baseline, and 6 and 18 months after randomization to determine the effects of MAU and FFT+MAU on youth offending, parenting and parent behaviour. Both groups showed large reductions in all measures of offending and antisocial behaviour, but there were no significant changes in parenting behaviour or the parent-child relationship over time.

There were also no significant differences between the intervention and control group at 6 or 18 months based on self-reported delinquency, police records of offending or symptoms or diagnoses of conduct disorders. These findings are in contrast to most previous trials of $\mathrm{FFT}^{1}$, which the researchers propose may be because MAU alone is effective at reducing antisocial behaviours.

Humayun,S., Herlitz, L., Chesnokov, M., Doolan, M., Landau, S. \& Scott, S. (2017), Randomized controlled trial of Functional Family Therapy for offending and antisocial behaviour in UK youth.

J Child Psychol Psychiatr. 58: 1023-1032. doi:10.1111/jcpp.12743

Further reading:

'Baldwin, S. et al. (2012) The effects of family therapies for adolescent delinquency and substance abuse: A meta-analysis. Journal of Marital Fam Ther. 38: 281-304. doi: 10.1111/j.1752-0606.2011.00248.x. 


\section{Children at risk of developing antisocial behaviours show deficits in affective empathy}

Researchers in the Netherlands and UK have monitored cardiovascular and electrodermal activity and eye tracking to assess affective and cognitive empathy in children at high risk of engaging in criminal behaviours. Children composing the high-risk group (114 boys aged 8-12 years) were recruited through the ongoing "Preventive Intervention Trajectory" study of younger siblings or children "of delinquents" and children failing primary school in Amsterdam, Netherlands. The high risk children, and a group of typically developing controls, were shown four different video clips: one neutral clip and three clips aiming to elicit emotions of fear, happiness or pain. During each clip, visual fixation to the video screen and attention to socially relevant cues (namely the eyes and faces of the actors) was determined by eye tracking and used as an indicator of social attention. Following the clips, the participants were asked to identify the type of emotion portrayed and its intensity to determine cognitive empathy. The researchers found no marked differences in these two parameters between the high risk and control groups. Heart rate and the skin conductance response (SCR) was also recorded during the presentation of each clip and used as a measure of affective empathy. Here, the high risk group had a lower heart rate when observing clips of pain and fear, and reduced SCR when observing pain. The researchers propose that specific deficits in affective empathy for negative emotions may have an important role in antisocial behaviour development. Going forward, they suggest that interventions should target affective empathy to promote pro-social behaviour.

Referring to:

van Zonneveld, L., Platje, E., de Sonneville, L., van Goozen, S. \& Swaab, H. (2017), Affective empathy, cognitive empathy and social attention in children at high risk of criminal behaviour. J Child Psychol Psychiatr. 58: 913-921. doi:10.1111/jcpp.12724
Glossary:

Cognitive empathy: the capacity of an individual to understand what others' emotions and thoughts might be, without being emotionally involved.

Affective empathy: the capacity of an individual to experience another person's feelings while understanding that they are distinct from one's own feelings.

Social attention: attention to socially relevant cues, in particular facially expressed cues, to gain information about the mental and emotional state of another person during social interaction.

Skin conductance response (SCR): a phenomenon whereby the skin momentarily becomes a better conductor of electricity when either external or internal stimuli occur that are physiologically arousing. Thus, the SCR is an objective, transient indication of autonomic nervous system arousal in response to a stimulus.

Pro-social behaviour: engagement in behaviours and/or acts that are considered to be generally beneficial or intended to help other people. Pro-social behaviours include feeling empathy or concern for others. 

Disruptive behavioural problems are a common psychopathology in children and adolescents, with an estimated global prevalence of $5.7 \%$ for any disruptive behaviour disorder ${ }^{1}$. The majority of intervention studies performed to date have focused on children residing in high-income, Western countries: whether the findings from these studies are transferable to other populations - namely low-income and middle-income countries (LMIC) - is unknown.

To bridge this knowledge gap, Professor Burkey and colleagues conducted a systematic review of randomized controlled trials to determine the efficacy of psychosocial interventions in preventing or reducing disruptive behaviour problems (namely oppositional defiant disorder and conduct disorder) in children ( $<18$ years) living in LMIC. They also evaluated the range and type of interventions for behaviour problems that have been assessed in children living in LMIC, and aimed to identify any issues with regards to intervention implementation and/ or dissemination.

The systematic review of 26 randomized controlled trials included 4,441 subjects and 28 active (non-pharmacological) psychosocial interventions that primarily targeted children, parents, families or teachers. Of these interventions, 15 were prevention interventions targeting general or at-risk populations and 13 were treatment interventions for those with behavioural problems. As predicted, the number of studies conducted in LMIC was low, with only $12 \%$ conducted in low-middle income and $31 \%$ conducted in low-income countries compared to $58 \%$ conducted in high-income countries. Geographic representation was restricted by only few studies conducted in Asia $(n=5)$ and the diversity of low-income countries overall.

The researchers found a significant reduction in disruptive behaviour problems across the prevention and treatment studies conducted in LMIC. Strong evidence was found for child-focused (including social skills training and cognitive behavioural therapy) and behavioural parent training interventions, suggesting that these strategies may be effective for children of all age and socioeconomic status groups. In addition, the use of group delivery models for most interventions and the requirement for non-specialist providers in $\sim 50 \%$ of the included studies supports their applicability to LMIC where resources are sparse.

Despite positive results, some limitations must be acknowledged. First, the researchers noted a high level of statistical heterogeneity in the intervention effects among the studies included in their meta-analysis. In addition, selection and performance biases were common in the studies included in the review. Despite these two limitations, the effects remained significant across multiple subgroup analyses, suggesting that many psychosocial interventions may be effective in reducing disruptive behavioural problems in children in LMIC.
In terms of prevention, no studies specifically evaluated a reduction in the incidence of behavioural disorders, which is considered a primary outcome in prevention research. In conclusion, Burkey and colleagues report strong support for the feasibility and effectiveness of prevention and treatment interventions to reduce disruptive behaviour problems in children in LMIC. The researchers propose that future research into the interventions for behaviour problems in LMIC need to follow a more rigorous study design, with in-depth reporting in the trials to accurately inform future implementation. With $>90 \%$ children living in LMIC, the researchers emphasize that implementing proven treatment interventions for disruptive behaviours in this population should be a global priority.

Referring to:

Burkey, M.D., Hosein, M., Morton, I., Purgato, M., Adi, A., Kurzrok., Kohrt, A.B. \& Tol, W.A. (2018), Psychosocial interventions for disruptive behaviour problems in children in low- and middle-income countries: a systematic review and meta-analysis. J Child Psychol Psychiatr, doi:10.1111/jcpp.12894

Further reading:

'Polanczyk, G.V. et al. (2015) Annual Research Review: A meta-analysis of the worldwide prevalence of mental disorders in children and adolescents. J Child Psychol Psychiatr, 56:345-365. doi:10.1111/jcpp.12381

\section{Glossary:}

Conduct disorder (CD): $C D$ is characterized by behaviour that violates either the rights of others or major societal norms. To be diagnosed with conduct disorder, symptoms must cause significant impairment in social, academic or occupational functioning. The disorder is typically diagnosed prior to adulthood.

Oppositional defiant disorder (ODD): ODD is characterised by a pattern of negativistic, hostile and defiant behaviour. The disturbance in behaviour causes clinically significant impairment in social, academic or occupational functioning and the behaviours do not occur exclusively during the course of a psychotic episode or mood disorder.

Behavioural parent training: an approach to treating child behaviour problems at home in which parents use taught procedures to alter interactions with their child, promote prosocial behaviour and decrease deviant behaviour.

Cognitive behavioural therapy: a form of talking therapy that encourages patients to manage their psycho-social problems by changing the way they think and behave; CBT focuses on current problems and finds practical ways to improve state-of-mind on a day-by-day basis.

Randomised controlled trial: an experimental setup whereby participants are randomly allocated to an intervention/treatment group or a control/placebo group; randomization of participants occurs after assessments for eligibility, and is used to minimize selection bias. 


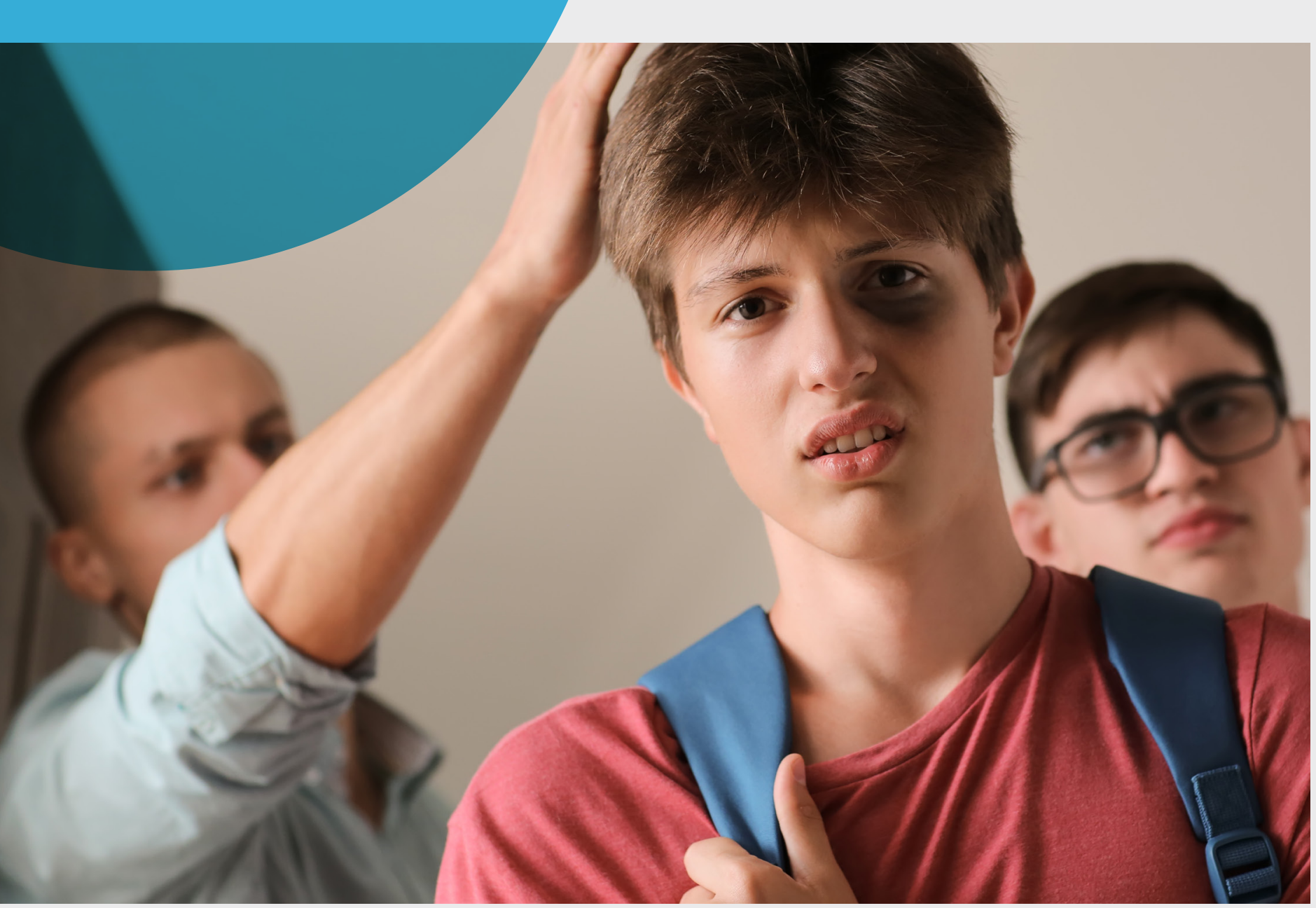

\section{Low empathy in adolescent boys predicts violent behaviour in adulthood}

Low empathy and low resting heart rate are established, independent risk factors of antisocial behaviour ${ }^{2}$. Now, researchers from the University of Pittsburgh have studied whether an interaction between these two factors during adolescence might mediate violent behaviour in early adulthood. The longitudinal study included 160 boys from low-income backgrounds. At age 12, the boys completed questionnaires to assess their disposition, empathy and pro-sociality (using the Children and Adolescent Dispositional Scale) and underwent electrocardiography to measure their resting heart rate.
At ages 17-20 years, the young men were assessed for characteristics of violent behaviour (using the SelfReport of Delinquency Questionnaire) and moral disengagement (using the Mechanisms of Moral Disengagement Scale). These measures were combined with juvenile court records collected between ages 15 and 18 years. Empathy alone negatively correlated with violent court petitions and moral disengagement whereas resting heart rate alone at age 12 was unrelated to all measures of violent behaviour. When testing for interactions between empathy and resting heart rate, however, the researchers found that low empathy predicted greater violence at age 20 only in men with a low resting heart rate. These findings suggest that resting heart rate and empathy interact in early adolescence to mediate violent behaviour later in life. The researchers propose that improving empathic skills in adolescents at risk for violent behaviour may be an effective preventative measure.

\section{Referring to:}

Galán, C.A., Choe, D.E., Forbes, E.E. \& Shaw, D.S. (2017), Interactions between empathy and resting heart rate in early adolescence predict violent behaviour in late adolescence and early adulthood. J Child Psychol Psychiatr. 58: 13701380. doi:10.1111/jcpp.12771

Further reading:

'Latvala, A., et al. (2015) A longitudinal study of resting heart rate and violent criminality in more than 700,000 men. JAMA Psychiatry, 72: 971-978.

${ }^{2}$ Knafo, A., et al. (2008) The developmental origins of a disposition toward

empathy: genetic and environmental contributions. Emotion, 8: 737. 


\section{Language impairment needs more recognition in the}

juvenile justice system

The prevalence of language and communication impairments in adolescents in custody is much higher than that seen in the general population, with estimates ranging from $60-90 \%$ compared to $7-12 \%$, respectively ${ }^{1}$. A study from Nathan Hughes and colleagues has now investigated co-morbidity of language difficulties in a cohort of 93 young male offenders (aged 15-18 years) held in a secure custodial facility in the UK. The participants completed a range of assessments to evaluate aspects of their communication, cognition and neurodevelopment: $47 \%$ demonstrated overall language skills markedly below the population average, of which $28 \%$ had overall language impairment. This latter group exhibited difficulties with communication and nonverbal cognition, and were at a higher risk of self-harm, depression and alcohol/substance misuse than those without impairment. Despite $>40 \%$ of those with language impairments previously being involved in the care system or attending a specialist school, the majority had not accessed any form of speech and language therapy prior to custody.

Further reading:

${ }^{1}$ Hughes, N. et al. (2012) Nobody made the connection; the prevalence of neurodisability in young people who offend. London:

Office for the Children's Commissioner.
These findings identify that access to speech and language services is severely limited in young offenders. The researchers conclude that there are likely to be many missed opportunities to identify language difficulties within the health, social care, and criminal justice and education systems. They propose that speech and language services should have a greater role in the youth justice system to facilitate early identification and support for those at risk of engaging in offending behaviour.

Referring to:

Hughes, N., Chitsabesan, P., Bryan, K., Borschmann, R., Swain, N., Lennox, C. \& Shaw, J. (2017), Language impairment and comorbid vulnerabilities among young people in custody. J Child Psychol Psychiatr. 58: 1106-1113. doi:10.1111/jcpp.12791 
Reconstructing child psychopathy into grandiose-manipulative,

callous emotional and daring-impulsive traits will facilitate diagnosis and treatment of conduct disorder

\section{By Dr. Jessica K. Edwards}


In 2017, Professor Randall Salekin at the University of Alabama, USA compiled a Research Review for the Journal of Child Psychology and Psychiatry that examined the main psychological and biological correlates of child psychopathy. Here, Professor Salekin outlines his key findings and explains that giving attention only to callous unemotional traits is an under-representation of psychopathy as a whole.

In 1941, Hervey Cleckley published a seminal paper describing a personality type characterized by low levels of empathy and sense of guilt, arrogance, superficial charm, and irresponsible and antisocial behaviours ${ }^{1}$. These characteristics formed the first description of adult psychopathy. Subsequent research in the 1990's broke psychopathy down into three dimensions: an interpersonal domain, consisting of grandiose-manipulative (GM) traits; an affective domain, consisting of callous unemotional (CU) traits; and a behavioural domain, consisting of daringimpulsive (DI) traits ${ }^{2}$.

The concept of child and adolescent psychopathy was born after adult psychopathy, when it was identified that the three dimensions (with the addition of antisocial conduct) were not unique to adults ${ }^{3-5}$. Importantly, and contrary to general assumption, psychopathic traits are modestly stable from early adolescence to young adulthood, and CU, GM and DI traits are all detectable very early in childhood ${ }^{6}$.

Despite the proposed multidimensional model for psychopathy, much research has continued to focus solely on CU traits, such as low levels of guilt, reduced empathy, callousness and uncaring behaviour. As a result, CU traits alone were added to the fifth revision of the Diagnostic and Statistical Manual of Mental Disorder (under the term "limited prosocial emotion") as a specifier for conduct disorder (CD) diagnosis, and the International Classification of Disease - 11th revision.

"The conception of this review was born out of a general dissatisfaction with research on $\mathrm{CU}$ traits alone and the implication that it was a sufficient index of psychopathy and as a specifier for CD", explains Salekin. "In addition, the lack of a good measurement for child psychopathic traits, and disconnect between child and adult research on psychopathy, led me to question what we really know about child psychopathic traits".
Salekin investigated the external cognitive, emotional, motivational, personality, parenting and biological correlates of child psychopathy. A striking overall finding was the extent of differences in the associations between the three child psychopathy dimensions and many psychological and biological variables.

For example, Salekin found that unlike CD, the broader construct of psychopathy is not associated with low IQ. However, when examining each of the three domains in isolation, he found that GM traits were more consistently associated with high IQ and sufficient perspective-taking, whereas CU traits were generally associated with low IQ and insufficient perspective-taking; DI traits had mixed associations with IQ. The available data for physiological correlates with the psychopathy construct are also mixed, with inconsistent associations between startle (nervous) responses (determined by electromyography, skin conductance response and heart rate) and GM, CU, and DI traits.

"It was surprising to find that the factors that undergird psychopathy can sometimes produce quite fractionated findings with a variety of external correlates", says Salekin. "This means that the processes that underlie the three dimensions could mean quite differentiate things in terms of heart rate variability and amplitudes or skin conductance to signalled (warning) and un-signalled (no warning) stimuli (like a shock or white noise) for the various specifiers of CD. Really, this does highlight how important it is to look at not only the broad construct but also the need to look at each dimension."

Somewhat more consistent data has, however, been reported for the associations between emotions, personality and parenting and the broader construct of psychopathy. Salekin found considerable support for an association between child psychopathy and an emotional deficit based on affect recognition, empathy, or distress cue responses. However, the specific nature of this emotional deficit remains unclear as too few studies have been conducted at the level of each individual dimension. For personality, the broad construct of child psychopathy seems to be associated with low conscientiousness, low agreeableness, reward responsiveness and social dominance. But once again, only a few studies have examined each psychopathy dimension. 
Salekin identified a surprising relationship between parenting and psychopathy dimensions: both harsh, inconsistent and cold parenting and supportive parenting may be involved in psychopathy trait development and/or maintenance. Specifically, youths with GM traits may have more supportive parents than youths with high CU traits, yet both groups of youths (and those with DI traits) engage in antisocial behaviour.

Finally, at the biological level, fMRI brain imaging studies have shown that GM traits may be less associated with functional brain impairments than CU or DI traits. Furthermore, structural, connectivity and functional neurological studies have all implicated the amygdalae in child psychopathy, which is in agreement with adult psychopathy. However, Salekin again acknowledges that although preliminary patterns are emerging between central nervous system functioning and psychopathy traits, the number of studies conducted and their sample sizes are too small to draw firm conclusions.

"Currently, there is still far too little known about the brain and how it operates with respect to psychiatric conditions and especially psychopathy, and we are not always clear about the regions of the brain implicated in deficits (lack of fear)", says Salekin. "This was recently pointed out by LeDoux ${ }^{7}$ who suggested that the fear circuitry needs to be reconsidered, noting that some of the fear processing likely occurs in the frontal cortex".
According to Salekin, a big issue that the field needs to make sense of relates to the processes that underlie each dimension and how they co-occur in a single configuration, with some facets masking the contents of other facets. For example, "how superficial charm and the generally polished outward surface of those with psychopathic traits can mask the emotionally devoid, and even emotionally deviant aspects of a psychopathic individual." Professor Salekin and his research team are actively trying to understand such important clinical observations as "the mask of sanity" ${ }^{1}$, alongside their ongoing research on the three dimensions of psychopathy in relation to CD.

Salekin expects that the largest gains in the field will likely be made if the research community examines the wider construct of psychopathy and its underlying dimensions, as well as their relation to $C D$, Oppositional Defiant Disorder (ODD) and in the future, adulthood antisocial personality disorder. In terms of diagnostic nomenclature and manuals, he hopes that the field will eventually make a notable step toward including the broader set of psychopathy dimensions as specifiers for CD. "It's difficult to deny that the sub-dimensions exist, and that they could better help understand the condition; their inclusion will help with clinical practice and guide clinicians in their work with kids with CD and potentially ODD", says Salekin.

"Better specification as to how CD manifests itself can only lead to better treatment and, thus, a quicker reduction in $\mathrm{CD}$ prevalence worldwide. Given that this disorder has a large toll on society, a large step is needed in this regard". Although further research is needed, Salekin hopes that the Proposed Specifiers for Conduct Disorder (PSCD) scale that he developed together with Professor Robert Hare will facilitate progress in this rea $^{8}$. Salekin also provides some initial thoughts on how the different dimensions might be included in diagnostic manuals to better specify CD?. 
In summary, Salekin's Research Review highlights that while some intriguing patterns are emerging, there are still too few studies examining psychopathy in children at the broad construct level and even fewer studies examining the three core dimensions of psychopathy. "There are key questions about whether we will move forward with this as one construct or whether it will be fractionated: I guess there is some risk that if we continue to simply focus on $\mathrm{CU}$ we will lose out on gathering important information about the other two dimensions and child psychopathy more broadly", poses Salekin. "This may also result in the child construct of psychopathy becoming largely separated from the work on adult psychopathy, yet there is a great deal of clinical history to speak to the importance of recognizing the broader condition".
Referring to:

Salekin, R.T. (2017), Research Review: What do we know about psychopathic traits in children? J Child Psychol Psychiatr. 58: 1180-1200. doi:10.1111/ jcpp. 12738

\section{Further reading:}

${ }^{1}$ Cleckley, H. The mask of sanity (5th edition) St Louis: Mosby; 1976.

${ }^{2}$ Hare R.D. (1991) The Hare Psychopathy Checklist-Revised (PCL-R). Toronto, ON, Canada: Multi-Health Systems.

${ }^{3}$ Forth, A.E. et al. (1990) Assessment of psychopathy in male young offenders. Psychol Assess. 2:342-344. doi: 10.1037/1040-3590.2.3.342.

4Frick, P.J. et al. (1994). Psychopathy and conduct problem in children. J Abnorm Psychol. 103:700-707. doi: 10.1037/0021-843X.103.4.700.

${ }^{5}$ Lynam D.R. (1997) Pursuing the psychopath: capturing the fledgling psychopath in a nomological net. J Abnorm Psychol. 106:425-438. doi: 10.1037/0021-843X.106.3.425.

${ }^{6}$ Assary, E. et al. (2015) Big-five and callous-unemotional traits in preschoolers. J Psychopathol Behav Assess. 37:371-379. doi: 10.1007/s10862014-9471-9

7LeDoux, J.E. (2014). Coming to terms with fear. Proc Natl Acad Sci U S A. 111: 2871-2878. doi: $0.1073 /$ pnas.1400335111

${ }^{8}$ Salekin, R. T. (2016). Psychopathy in childhood: Toward better informing the DSM-5 and ICD-11 conduct disorder specifiers. Personality Disorders: Theory, Research, and Treatment. 7:180-191. doi: 10.1037/peroooo150

9Salekin, R. T. (2016). Psychopathy in childhood: Why should we care about grandiose-manipulative and daring-impulsive traits? British Journal of Psychiatry, 209, 189-191. doi: 10.1192/bjp.bp.115.179051
Glossary:

Grandiose-manipulative (GM) traits: a dimension of psychopathy in which an affected individual displays grandiosity, arrogance, dishonest charm and manipulation.

Callous unemotional (CU) traits: a dimension of psychopathy in which an affected individual displays low empathy, low guilt and no remorse.

Daring-impulsive (DI) traits: a dimension of psychopathy in which an affected individual exhibits risk-taking and thrill-seeking behaviour.

Conduct disorder (CD): $C D$ is characterized by behaviour that violates either the rights of others or major societal norms. To be diagnosed with conduct disorder, symptoms must cause significant impairment in social, academic or occupational functioning. The disorder is typically diagnosed prior to adulthood.

Perspective taking: the ability to take someone else's viewpoint into account when thinking.

Oppositional defiant disorder (ODD): ODD is characterised by a pattern of negativistic, hostile and defiant behaviour. The disturbance in behaviour causes clinically significant impairment in social, academic or occupational functioning and the behaviours do not occur exclusively during the course of a psychotic episode or mood disorder.

Antisocial personality disorder (APD): APD is characterised by a pervasive pattern of disregard for and violation of the rights of others occurring since age 15 years. The affected individual is at least age 18 years, has shown signs of conduct disorder with onset before age 15 years and the occurrence of antisocial behaviour is not exclusively during the course of schizophrenia or a manic episode.

Affect recognition: recognition of emotion (fear or distress) in others by interpreting facial or vocal expressions.

Empathy: the ability to understand another person's thoughts, feelings and condition from their point of view.

Distress cue response: recognition of distress in the faces of victims. 


\section{Police contact during adolescence can delay female psychosocial maturation}

Over the past 30 years, the rate of female juvenile arrests in the USA has almost doubled. Despite this rapid increase, most research into juvenile delinquency has primarily focused on males. To determine some of the driving factors of female juvenile delinquency, Alison Hipwell and colleagues assessed the number of police contacts and arrests, and levels of self-control, personal responsibility and delinquency annually from age 12 to 17 years in a population sample of 2,450 girls enrolled in the Pittsburgh Girls Study. At age 12, 5.3\% girls had been involved in illegal activity; this rate peaked at $12.3 \%$ at age 15 years. Interestingly, the levels of self control and personal responsibility at age 12 were lower in those who had had police contact than those without police contact. Regression analysis found that year-to-year increases in self-control or personal responsibility predicted a reduced likelihood of police contact. In the reverse association, an instance of police contact or arrest predicted decreases in self-control and personal responsibility in the following year. These data suggest for the first time, that police contact and/or police arrests may be associated with delayed adolescent psychosocial maturation in girls. The researchers propose that programs that support growth in self-control and responsibility in girls will help prevent future justice system involvement.

Referring to:

Hipwell, A.E., Beeney, J., Ye, F., Gebreselassie, S.H., Stalter, M.R., Ganesh, D., Keenan, K. \& Stepp, S.D. (2018), Police contacts, arrests and decreasing self-control and personal responsibility among female adolescents. J Child Psychol Psychiatr. doi:10.1111/jcpp.12914

\section{Glossary:}

Regression analysis: a statistical method to model the relationship between a dependent (response) variable and one or more independent (predictor) variables. 


\section{Making Child and Family Therapies More Effective}

\section{Inaugural Judy Dunn National Conference}

Friday 9 November 2018, 09.00 - 17.00

Royal Northern College of Music, Manchester

About the day

ACAMH is pleased to announce the launch of the Judy Dunn National Conference. In light of recent attention on the importance of a holistic approach to good mental health, the inaugural event will be focusing on psychological interventions for both children and their families.

Topics

- Interventions for adopted and fostered children and their families/carers

- Working with goals across therapies with children and young people

- Results and implications of an experimental trial focusing on the therapeutic alliance

- Whole school approaches to mental health

- Psychological interventions to improve child outcomes for mentally ill mothers

Speakers

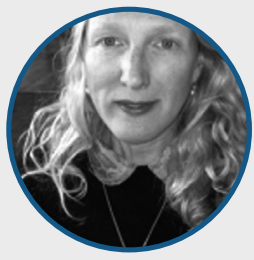

Keynote

Professor Kathryn Abel Professor of

Psychological Medicine and Reproductive

Psychiatry, University of Manchester

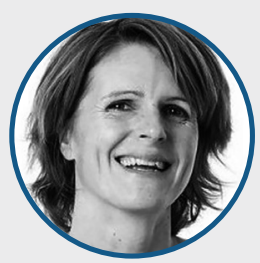

Dr Sophie Browning Consultant Clinical Psychologist

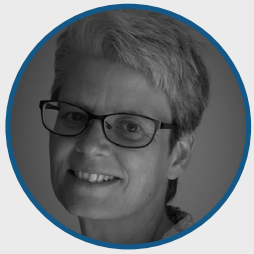

Dr. Carol Taylor Research Associate Division of Neuroscience \& Experimental Manchester

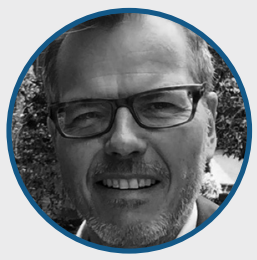

Dr Duncan Law Consultant Clinical Psychologist, Anna Freud Centre Psychology, University of

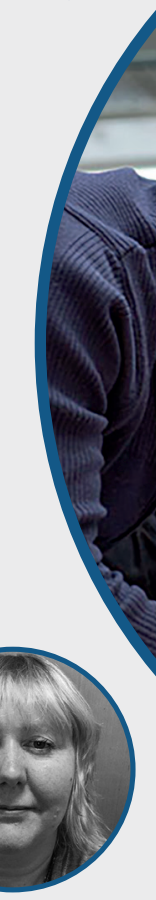

Dr Rachel Elvins Consultant Child and Adolescent Psychiatrist

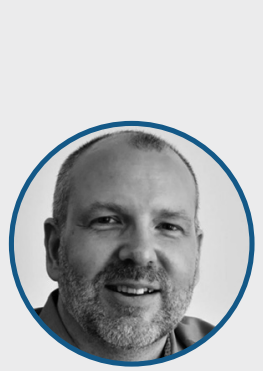

Dr Matt Woolgar Consultant Clinical Psychologist, SLaM

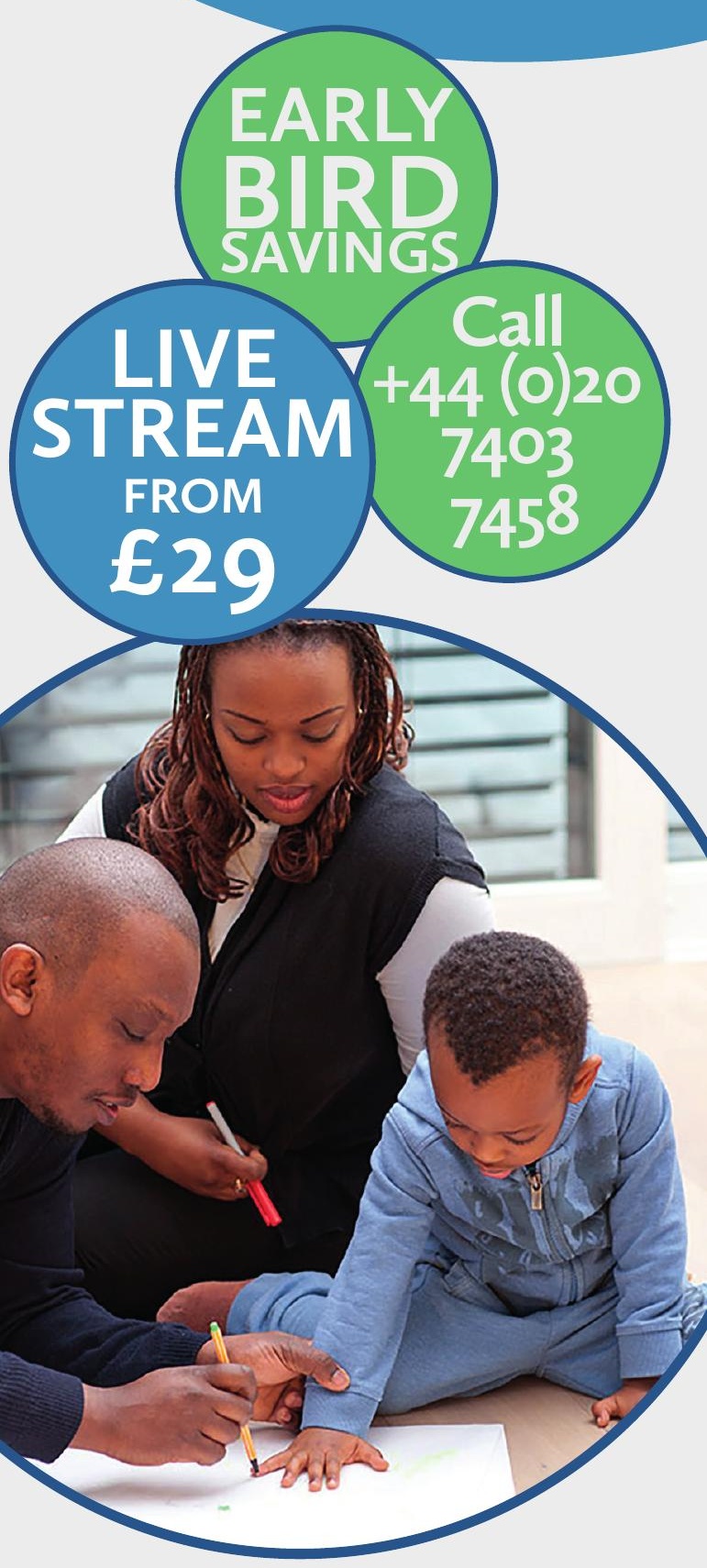

BOOK NOW ONLINE or call 02074037458

LIVE STREAM

E29pp Member

Ł69pp Non-Member

IN PERSON

PLATINUM: $€ 179$

GOLD: $€ 205$

SILVER/BRONZE: $€ 231$

BRONZE: $€ 231$

DIGITAL/PUBLICATIONS: $£ 99$

Non-Members £257 